\title{
Methods for Accurate Homology Modeling by Global Optimization
}

Keehyoung Joo, Jinwoo Lee, Jooyoung Lee

\begin{abstract}
High accuracy protein modeling from its sequence information is an important step toward revealing the sequence-structure-function relationship of proteins and nowadays it becomes increasingly more useful for practical purposes such as in drug discovery and in protein design. We have developed a protocol for protein structure prediction that can generate highly accurate protein models in terms of backbone structure, side-chain orientation, hydrogen bonding and binding sites of ligands. In order to obtain accurate protein models, we have combined a powerful global optimization method with traditional homology modeling procedures such as multiple sequence alignment, chain building and side-chain re-modeling. We have built a series of specific score functions for these steps, and optimized them by utilizing conformational space annealing, which is one of the most successful combinatorial optimization algorithms currently available.
\end{abstract}

Key words: Homology modeling; protein structure prediction; global optimization; energy function; multiple sequence alignment; side-chain modeling; conformational space annealing

Keehyoung Joo

School of Computational Sciences, Korea Institute for Advanced Study, Seoul 130-722, Korea, e-mail: newton@kias.re.kr

Jinwoo Lee

Department of Mathematics, Kwangwoon University, Seoul 139-701, Korea, e-mail: jinwoolee@kw.ac.kr

Jooyoung Lee

School of Computational Sciences, Korea Institute for Advanced Study, Seoul 130-722, Korea, e-mail: jlee@kias.re.kr 


\section{Introduction}

Recently, protein structure prediction by homology modeling has become a basic tool that is routinely used in structural biology and bioinformatics [2, 19]. Although many computational methods have been developed in this field, high accuracy protein modeling still remains as a challenging problem. For example, it is rather difficult to generate protein models which are more accurate than what one can get by simply copying the best available homologus protein (out of the templates used for homology modeling).

In the recent CASP experiments (CASP7 and CASP8) for protein structure prediction, the high-accuracy template based modeling (HA-TBM) category is considered separately along with template based modeling (TBM) and free modeling (FM) categories, and there were many examples where protein models were more accurate than the best available templates in terms of accuracies of backbone structure, side-chain orientation, hydrogen bonding and usefulness for molecular replacement in X-ray crystallography $[17,11]$.

Three major steps of the standard homology modeling protocol are multiple sequence alignment, 3D (three dimensional) model building and side-chain remodeling, and recently, we have incorporated the global optimization method called conformational space annealing (CSA) to these three procedures to generate highly accurate protein models. In detail, the protocol of homology modeling using CSA consists of the following five steps: 1) fold recognition (finding homologus templates from known protein structures) 2) multiple sequence/structure alignment by global optimization 3) 3D structure modeling 4) assessment of protein models and alignments 5) side-chain remodeling by global optimization.

Fold recognition is to find homologus templates to the target protein from known protein structures in the PDB, and this step of identifying similar structures in the $\mathrm{PDB}$ is the most crucial one for successful homology modeling. Many sequencebased fold recognition methods incorporate properties of sequence similarity, profile similarity, and secondary structure similarity between proteins. Often, multiple templates are obtained by fold recognition, and the next step is to extract as much useful structural information from them, typically by performing multiple alignment between the target protein and templates.

In the second step, in order to generate more useful multiple sequence alignments (MSAs), we developed a method, called MSACSA, which explores the diverse alignment space to search rigorously low-energy alignments of given templates based on a consistency-based score function [10]. In the following steps, we generate many candidate alignments, and construct initial 3D models using MODELLER, and assess the quality of the alignments by assessing those of the 3D models by using a support vector regression machine. Here, preferred combinations of templates as well as choices for multiple alignment out of many alternative solutions are determined. For 3D model building from a few selected alignments, we optimize the MODELLER energy function as rigorously as possible to generate protein structures satisfying as much spatial restraints derived from its alignment as well as proper stereochemistry of proteins [12]. For side-chain remodeling, again we 
adopt the global optimization method of CSA to determine the orientations of sidechains both in the surface and inside the core area of protein structures [11]. Here the backbone-dependent rotamer library of SCWRL 3.0 is used. Below, we describe each step of the protocol to generate highly accurate protein models by global optimization.

\section{Materials and Methods}

For protein structure modeling, various bioinformatics and 3D modeling-related tools should be first installed in your computer system. They include PSI-BLAST, PSIPRED, MODELLER, the backbone-dependent rotamer library of SCWRL 3.0, DFIRE, DSSP, TM-align and SPICKER. PSI-BLAST program is a basic tool to generate sequence profile by searching protein sequence databases (e.g. $n r$ database from NCBI) [1]. Secondary structure of a protein sequence is predicted by PSIPRED [9]. MODELLER is a 3D structure building program by using templates and an alignment as inputs [19]. The backbone-dependent rotamer library of SCWRL 3.0 program [4] can be downloaded from Dr. Dunbrack's webpage ${ }^{1}$. DFIRE, an energy function to assess the quality of a given protein structure can be obtained by email request to the authors [22]. DSSP program calculates secondary structures, solvent accessibility, and other structural properties for a given protein 3D structure [13]. TM-align calculates structural similarity for two given protein structures and, SPICKER is a clustering program to select a few representative structures from many $(\sim 100)$ predicted models.

For optimization of energy functions for multiple sequence alignment and 3D model building, parallel computing resources are recommended to reduce computation time, and parallel algorithms of conformational space annealing method have to be implemented on a parallel computing system (e.g. a cluster system). A few implementations of CSA can be found from the literature $[16,15]$ and a recent CHARMM package containing the CSA routine, which will be available soon [3].

\section{Fold recognition}

Fold recognition is the starting point of homology modeling. We have used an inhouse profile-profile comparing method, called FOLDFINDER to rank templates of known structures from PDB [11]. We have built a profile database of protein chains by using PSI-BLAST with standard parameters (E-value cutoff is set to 0.0001 and the procedure is iterated 3 times). For example, for CASP7 experiment, we built a profile database of 11914 chains obtained from PISCES culling server [21] at 95\% sequence identity level with sequence length in the range of 50 1000 residues.

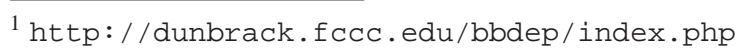


11914 chains include X-ray and NMR structures but not EM structures. We also built secondary structure profiles for chains in the database by using DSSP program (coil, helix and extended states are represented by vectors $(1,0,0),(0,1,0)$, and $(0,0,1)$, respectively).

1. For each chain in the database, its pair-wise sequence alignment with the target sequence is obtained by dynamic programing using the following match score: $S_{i j}=S_{i j}^{p}+0.4 * S_{i j}^{h}+0.01$, where $S_{i j}^{p}$ is the pearson's correlation coefficient between the $i$-th row vector of the target sequence profile and the $j$-th row vector of the template profile. $S_{i j}^{h}$ is the pearson's correlation coefficient between the $i$-th row vector of the predicted secondary structure probability by PSIPRED and the $j$-th row vector of the secondary structure profile of the template. Dynamic programming is performed using the affine gap penalty function of $w(k)=-(1.5+0.07 * k)$ where $k$ is the gap length. ${ }^{2}$ End-gaps are not penalized (global-local alignment).

2. All template chains of the database are sorted according to their alignment scores, and the statistical significance of an alignment score is measured by its Z-score and p-value. An example of the FOLDFINDER output is shown in Fig. 1.

3. Considering top-scoring templates with z-score typically greater than 4.0, structurally redundant templates $(\mathrm{TM}$-score $>0.98)$ are removed. With these templates, we further perform structural clustering by using TMalign considering all pairs of templates. We consider a subset of templates where TM-score $<0.5$ between all members. We prepare typically 5 to 10 sets of template combinations. Each combination is called a list and it is used as an input to the subsequent step of multiple alignment. In the CASP experiments, the number of templates ranges 1 to 15 for one list.

\section{Multiple sequence/structure alignment}

We perform multiple sequence/structure alignment by using MSACSA method [10]. For each list of template combination, we execute the following steps to obtain lowenergy multiple alignments by CSA optimization. Optimization by CSA is repetedly applied in this chapter and a brief description of the major steps of CSA is provided in appendix.

1. Preparation of pair-wise restraint library: For each template in the list, we carry out profile-profile alignment with the target sequence using FOLDFINDER as described in the fold recognition step. Matched residue pairs are stored into the pair-wise restraint library. In addition, for all pairs of templates in the list, pairwise structure alignment is carried out using TM-align, and the matched residue pairs are also added into the pair-wise restraint library. For each residue pair in

2 Parameters were obtained by optimizing the average accuracy of sequence alignments for 388 references with sequence identity $\leq 40 \%$ from HOMSTRAD datbase. 
the restraint library, the sequence identity between two sequences to which the two residues belong is assigned as the weight $w$ to be used in the score function below.

2. We define an energy function for a given multiple alignment $A$, as the measure of consistency of $A$ with the restraint library. With $N$ sequences and $M$ aligned columns, it becomes:

$$
E(A)=-100 \times \frac{\sum_{i, j=1, i<j}^{N} w_{i j} \sum_{k=1}^{M} \delta_{i j}^{k}(A)}{\sum_{i, j=1, i<j}^{N} w_{i j} L_{i j}},
$$

where $\delta_{i j}^{k}(A)=1$ if the aligned residues between the $i$-th and the $j$-th sequences at the $k$-th column are in the library, otherwise $\delta_{i j}^{k}(A)=0 . L_{i j}$ and $w_{i j}$ are the pairwise alignment length and the sequence identity between the $i$-th and the $j$-th sequences, respectively.

3. Define the distance measure between two given multiple alignments as the number of residue mismatches considering all pairwise sequence alignments between the two given multiple alignments.

4. Local optimization to minimize the energy value of a given multiple alignment is carried out by a series of perturbation of the alignment for up to $t$ times. Typically, we set $t=10 N L_{\max }$, where $L_{\max }$ is the length of the largest sequence in the list. Perturbations are performed by local moves of gaps in the alignment. ${ }^{3}$

5. Combination of two multiple alignments: we generate a daughter alignment by replacing a part of a seed alignment by the corresponding part of another alignment. We limit the replacing part within $40 \%$ of the seed alignment.

6. With the preparation steps of 3,4 and 5, it is straightforward to carry out CSA to optimize $E(A)$ defined in eq. (1) to generate a total of 100 multiple alignments.

An example of the lowest-energy alignment and the energy landscape of the multiple alignment are shown in Fig. 2. This step is the key process for modeling highly accurate protein 3D structures. A total of 100 multiple sequence alignments obtained from this step for each list of templates are used as the input for the next step.

\section{Assessment of alignment/3D structure modeling}

In this step, we select 5 to 10 alignments by applying an assesment method. The assement is carried by a machine trained by support vector regression for feature vectors which are extracted from 3D protein models generated by MODELLER. Details of the assesment method is described in the appendix 'Model validation'. Selected alignments are used to generate higher quality 3D protein models by applying CSA method to optimize the MODELLER energy function [12].

\footnotetext{
3 These moves consist of random insertion, deletion, and relocation of gap(s) [8, 14].
} 
1. For the assessment of an alignment, we first generate 25 protein $3 \mathrm{D}$ models using MODELLER and the alignment under evaluation.

2. The quality of each $3 \mathrm{D}$ model is evaluated using the assesment method, and the quality of each alignment is estimated by the average 3D model quality from 25 initial models.

3. 5 to 10 top alignments are selected to proceed with the subsequent procedures.

4. For each alignment selected, we generate 100 protein 3D models by further optimization of MODELLER energy function using the CSA method, which we call as MODELLERCSA [12].

5. To execute MODELLERCSA, one needs to provide a few preliminary procedures: distance measure between two protein 3D models is defined as the $C_{\alpha}$ RMSD value between them. For local energy minimization, we used what is already implemented in the MODELLER package (conjugate-gradient minimization method). To generate a daughter model by crossover, we replace a part of the seed model by the corresponding part of another model. The replacement is limited up to $40 \%$ of the seed model as before. (See Note 3 )

It is shown [12] that the quality of a protein 3D model improves as its MODELLER energy is optimized. The comparison of 3D model qualities between structures generated by MODELLER and MODELLERCSA is shown in Fig. 3. Backbone accuracies as well as side-chain accuracies are plotted in terms of the MODELLER energy. Five representative models among 100 optmized models are selected by re-assesment of the models and clustering them into five groups. These five models are used for side-chain remodeling in the next procedure.

6. By using the same assesment method used above, we select top alignments and five models generated by MODELLERCSA.

7. By using SPICKER clustering method, we select representative models from cluster centers. Typically, we select a total of five models.

\section{Side-chain modeling}

We have used the backbone-dependent rotamer library of SCWRL 3.0 [4] to remodel side-chains of a given protein 3D model. For each 3D model selected from the previous step, we have built a target-specific rotamer library based on the consistency of the side chain conformations:

1. For each residue $i$, we calculate the average $\left(m_{i}\right)$ and the standard deviation $\left(\sigma_{i}\right)$ of $\chi_{i}^{1}$ angles of 100 models.

2. If $\sigma_{i} \leq 15^{\circ}$, we add 10 sets of all $\chi_{i}^{1}$ angles closest to $m_{i}$ into the rotamer library.

3. If $\sigma_{i}>15^{\circ}$, we use the backbone-dependent rotamer library SCWRL 3.0 for the residue. 
Rotamers are optimized by CSA, which is called ROTAMERCSA, to remodel side-chains of a selected model using the rotamer library and the energy function below.

4. An energy function $E$ is defined for side-chain optimization: $E=E_{S C W R L}+$ $E_{\text {DFIRE }}$ where $E_{S C W R L}$ is the score function used in SCWRL 3.0 and $E_{\text {DFIRE }}$ is the DFIRE energy [22].

5. Distance measure between two sets of side-chain conformations are defined as the sum of Euclidean distance for corresponding rotamer angles.

6. Local minimization is carried out by stochastic quenching as in the case of MSACSA.

7. A daughter conformation is generated by replacing a part of seed model's rotamers by the corresponding part of another model's rotamers.

8. Now, run CSA.

Fig. 4 shows side-chain accuracies of 27 HA-TBM targets from CASP7 obtained by ROTAMERCSA. Results by MODELLER as well as MODELLERCSA are also shown for comparison. It illustrates step-by-step improvement of the side-chain modeling. An example of the final 3D model after side-chain remodeling is shown in Fig. 5.

\section{Notes}

1. In the fold recognition step, when the top scoring template by FOLDFINDER is not so promineat in terms of $\mathrm{z}$-score ( $\mathrm{z}$-score $<3.0$ ), additional template candidates by other methods are also considered. Other fold recognition web servers include 3D-jury (http://bioinfo.pl/ 3djury) [7] and HHsearch [20] provided from web server.

2. Selecting templates should be carefully considered in aspects of alignment length, sequence identity, and consistency of secondary structure between target and templates. Also, if there are gap regions especially in the target sequence of multiple alignment, It is good to consider templates which can cover gap regions in the alignment.

3. In the MODELLERCSA, a daughter model is combined by using internal variables of two parent 3D models (such as bond angles, bond length and dihedral angles). A consecutive part of one parent's internal coordinates are replaced by the corresponding internal coordinates of the other parent, and resulting structure is subject to subsequent energy minimization. As a result, daughter structures partially inherit bond angles, bond lengths, and backbone and side-chain dihedral angels of their parents.

4. SPICKER uses distance cut value of $3.5 \AA$ for clustering. We have used a variable distance cut value in the range $1.0 \sim 3.5 \AA$.

5. Accuracies of side-chain for target solved in NMR experiment are relatively lower than solved in X-ray crystallography. 
6. The value of $D_{\text {cut }}$ is kept constant after it reaches $D_{\text {ave }} / 5$.

\section{Appendix}

\section{A brief description of conformational space annealing (CSA)}

Recently, CSA method is implemented in CHARMM, and the source code of CSA is available [3]. The CSA method searches the whole conformational space in its early stages and narrows the search to smaller regions with low energy as the distance cutoff, $D_{\text {cut }}$, which defines a (varying) threshold for the similarity between two solutions, is reduced. As in genetic algorithms, it starts with a preassigned number (50 in this work) of randomly generated and subsequently energy-minimized solutions. This pool of solutions/conformations is called the bank. At the beginning, the bank is a sparse representation of the entire conformational space. In the following, the meaning of conformation depends on the context where CSA is used. For multiple sequence alignment optimization, a conformation means an alignment. For 3D structure modeling, it presents a protein 3D structure model, and for side-chain remodeling, it refers to a set of side-chain conformations for a given fixed backbone structure. For implementation of CSA, we need a serise of new concepts. They are (i) an energy function to minimize, (ii) a distance measure between two conformations, (iii) a local minimizer of a given conformation, (iv) ways to combine two parent conformations to generate a daughter one. For details, see each section of the methods. Equipped with these four concepts, CSA proceeds as follows:

1. Generate 50 conformations which are randomly generated and subsequently energy minimized by a local minimizer.

2. Calculate $D_{\text {ave }}$ as the average distance between all pairs of the 50 conformations, and set $D_{\text {cut }}$ as $D_{\text {ave }} / 2$.

3. Select 30 distinct conformations called seeds which have not yet been used.

4. For each seed, perturb the conformation and subsequently energy minimize the perturbed conformation to generate a daughter conformation. If we generate 20 daughter conformations per seed, a total of $30 \times 20=600$ daughter conformations are prepared.

5. Update the existing 50 conformations using the 600 daughters by a special update scheme as described below.

6. Reduce $D_{\text {cut }}$ by a fixed ratio $r=0.997$ (see Note 6).

7. Go to the seed selection step until all seeds are used.

8. When all seeds are used, one iteration is completed. Set all conformations as unused, and repeat another iteration of the search.

9. If the second iteration completes, and the number of the pool is not 100 , add additional 50 random and subsequently energy-minimized conformations to the pool. Set $D_{\text {cut }}=D_{\text {ave }} / 2$, and go to the seed selection step once again. If the second iteration completes, and the number of pool is 100, it completes the CSA. 
Energy minimization: For continuous function with gradient available, conjugate gradient minimization is used. For a discrete function to optimize as in the case of multiple alignment and side-chain remodeling, we used a quench procedure as follows. Perturb a conformation and compare its energy with original one, and take the lower energy one. Repeat this process by a fixed number of trials.

Update scheme: For each daughter conformation, $\alpha$, the closest conformation A in terms of the corresponding distance measure (see each section of the methods) is determined. Let us denote the distance as $D(\alpha, A)$. If $d(\alpha, A) \leq D_{\text {cut }}, \alpha$ is considered similar to $\mathrm{A}$; in this case $\alpha$ replaces $\mathrm{A}$ in the pool of conformations provided that it is lower in energy. If $\alpha$ is not similar to A, but its energy is lower than that of the highest-energy conformation in the bank, B, $\alpha$ replaces B. In neither of the above conditions holds, $\alpha$ is rejected.

\section{Model validation}

In order to assess the quality of a given 3D model, you should build a support vector regression (SVR) machine using the following four steps.

1. Prepare a set of decoy structures with known structural quality in terms of TMscore.

2. For each model, calculate the following five feature components. In the following, $N_{\text {res }}$ is the number of residues of the given model.

a. $S S_{\text {score }}=-\sum_{i=1}^{N_{\text {res }}} P(\operatorname{SSTYPE}(i))$, where $P(\cdot)$ is the probability value from PSIPRED, and SSTYPE $(i)$ is the secondary structure type of the $i$-th residue.

b. $S A_{\text {score }}=\sum_{k=1}^{25} \sum_{i=1}^{N_{\text {res }}} D_{k}(i)\left(R S A_{\text {model }}(i)-R S A_{k}(i)\right)^{2}$, where $D_{k}(i)$ is the weighted Euclidean distance between profiles from the query and the $k$-th nearest neighbor in the database, $R S A_{\text {model }}(i)$ is the relative solvent accessible surface area(SASA) of the $i$-th residue of the model, and $R S A_{k}(i)$ is the relative SASA of the $i$-th residue of the $k$-th neighbor.

c. $H P_{\text {score }}=\sum_{i=1}^{N_{\text {res }}} \operatorname{DsspACC}(i) * H P(i)$, where $\operatorname{DsspACC}(i)$ is the SASA of residue $i$ from DSSP, and $H P(i)$ is the HP-table value for the $i$-th residue.

d. DFIRE energy of the model.

e. Modeller energy of the model.

3. We are now prepared with a table which contains TM-scores and five feature components for all decoy structures.

4. Build a support vector regression machine using the table by LIBSVM $[6,5]$.

Now you can predict TM-score of a given model by SVR machine using following procedure:

${ }^{4}$ We have used the hydrophobicity values of $0.74,0.91,0.62,0.62,0.88,0.72,0.78,0.88,0.52$, $0.85,0.85,0.63,0.64,0.62,0.64,0.66,0.70,0.86,0.85,0.76$ for residue types $\mathrm{A}, \mathrm{C}, \mathrm{D}, \mathrm{E}, \mathrm{F}, \mathrm{G}, \mathrm{H}$, I, K, L, M, N, P, Q, R, S, T, V, W, Y [18]. 
5. For a given model, calculate the five feature components described above.

6. Predict TM-score of the given model using the pre-built support vector regression machine.

7. For each template combination, we assign the quality of the list/alignment by the average of the predicted TM-scores of the 3D models.

\section{Acknowledgments}

This work was supported by Creative Research Initiatives (Center for in silico Protein Science, 2009-0063610) of MEST/KOSEF.

\section{References}

1. Altschul, S.F., Madden, T.L., Schaffer, A.A., Zhang, J., Zhang, Z., Miller, W., Lipman, D.J.: Gapped blast and psi-blast: a new generation of protein database search programs. Nucleic Acids Res 25(17), 3389-402 (1997)

2. Baker, D., Sali, A.: Protein structure prediction and structural genomics. Science 294(5540), 93-96 (2001)

3. Brooks, B.R., Bruccoleri, R.E., Olafson, B.D., States, D.J., Swaminathan, S., Karplus, M.: Charmm: A program for macromolecular energy, minimization, and dynamics calculations. Journal of Computational Chemistry 4(2), 187-217 (1983)

4. Canutescu, A.A., Shelenkov, A.A., Dunbrack, R.L.: A graph-theory algorithm for rapid protein side-chain prediction. Protein Sci 12(9), 2001-2014 (2003)

5. Chang, C.C., Lin, C.J.: LIBSVM: a library for support vector machines (2001). Software available at http://www.csie.ntu.edu.tw/ Cjlin/libsvm

6. Fan, R.E., Chen, P.H., Lin, C.J.: Working set selection using second order information for training support vector machines. J. Mach. Learn. Res. 6, 1889-1918 (2005)

7. Ginalski, K., Elofsson, A., Fischer, D., Rychlewski, L.: 3d-jury: a simple approach to improve protein structure predictions. Bioinformatics 19(8), 1015-1018 (2003)

8. Ishikawa, M., Toya, T., Hoshida, M., Nitta, K., Ogiwara, A., Kanehisa, M.: Multiple sequence alignment by parallel simulated annealing. Comput Appl Biosci 9(3), 267-73 (1993)

9. Jones, D.T.: Protein secondary structure prediction based on position-specific scoring matrices1. Journal of Molecular Biology 292(2), 195-202 (1999)

10. Joo, K., Lee, J., Kim, I., Lee, S.J., Lee, J.: Multiple sequence alignment by conformational space annealing. Biophys. J. 95(10), 4813-4819 (2008)

11. Joo, K., Lee, J., Lee, S., Seo, J.H., Lee, S.J., Lee, J.: High accuracy template based modeling by global optimization. Proteins 69 Suppl 8(9999), 83-89 (2007)

12. Joo, K., Lee, J., Seo, J.H., Lee, K., Kim, B.G., Lee, J.: All-atom chain-building by optimizing modeller energy function using conformational space annealing. Proteins $\mathbf{7 5}, 1010-1023$ (2009)

13. Kabsch, W., Sander, C.: Dictionary of protein secondary structure: pattern recognition of hydrogen-bonded and geometrical features. Biopolymers 22(12), 2577-2637 (1983)

14. Kim, J., Pramanik, S., Chung, M.J.: Multiple sequence alignment using simulated annealing. Comput Appl Biosci 10(4), 419-26 (1994)

15. Lee, J., Lee, I.H., Lee, J.: Unbiased global optimization of lennard-jones clusters for $\mathrm{n} \leq 201$ using the conformational space annealing method. Phys. Rev. Lett. 91(8) (2003) 
16. Lee, J., Scheraga, H.A., Rackovsky, S.: New optimization method for conformational energy calculations on polypeptides: Conformational space annealing. J. Comput. Chem. 18(9), 1222-1232 (1997)

17. Read, R.J., Chavali, G.: Assessment of casp7 predictions in the high accuracy template-based modeling category. Proteins 69 Suppl 8, 27-37 (2007)

18. Rose, G.D., Geselowitz, A.R., Lesser, G.J., Lee, R.H., Zehfus, M.H.: Hydrophobicity of amino acid residues in globular proteins. Science 229(4716), 834-838 (1985)

19. Sali, A., Blundell, T.L.: Comparative protein modelling by satisfaction of spatial restraints. J Mol Biol 234(3), 779-815 (1993)

20. Söding, J.: Protein homology detection by hmm-hmm comparison. Bioinformatics 21(7), 951-960 (2005)

21. Wang, G., Dunbrack, R.L.: Pisces: recent improvements to a pdb sequence culling server. Nucleic Acids Res 33(Web Server issue) (2005)

22. Zhou, H., Zhou, Y.: Distance-scaled, finite ideal-gas reference state improves structure-derived potentials of mean force for structure selection and stability prediction. Protein Sci 11(11), 2714-2726 (2002) 
Fig. 1 An example of the FOLDFINDER output for the target T0506 of CASP8 experiment is shown. Templates with z-score $>4.0$ are considered to be significant hits for a target sequence.

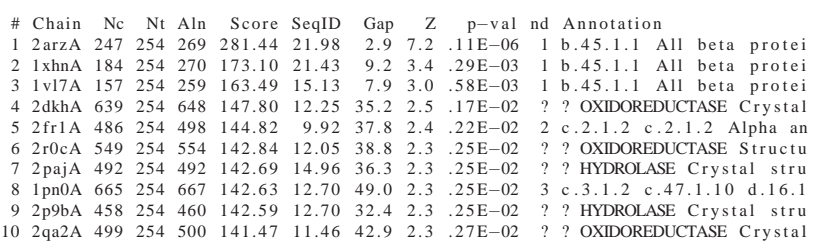



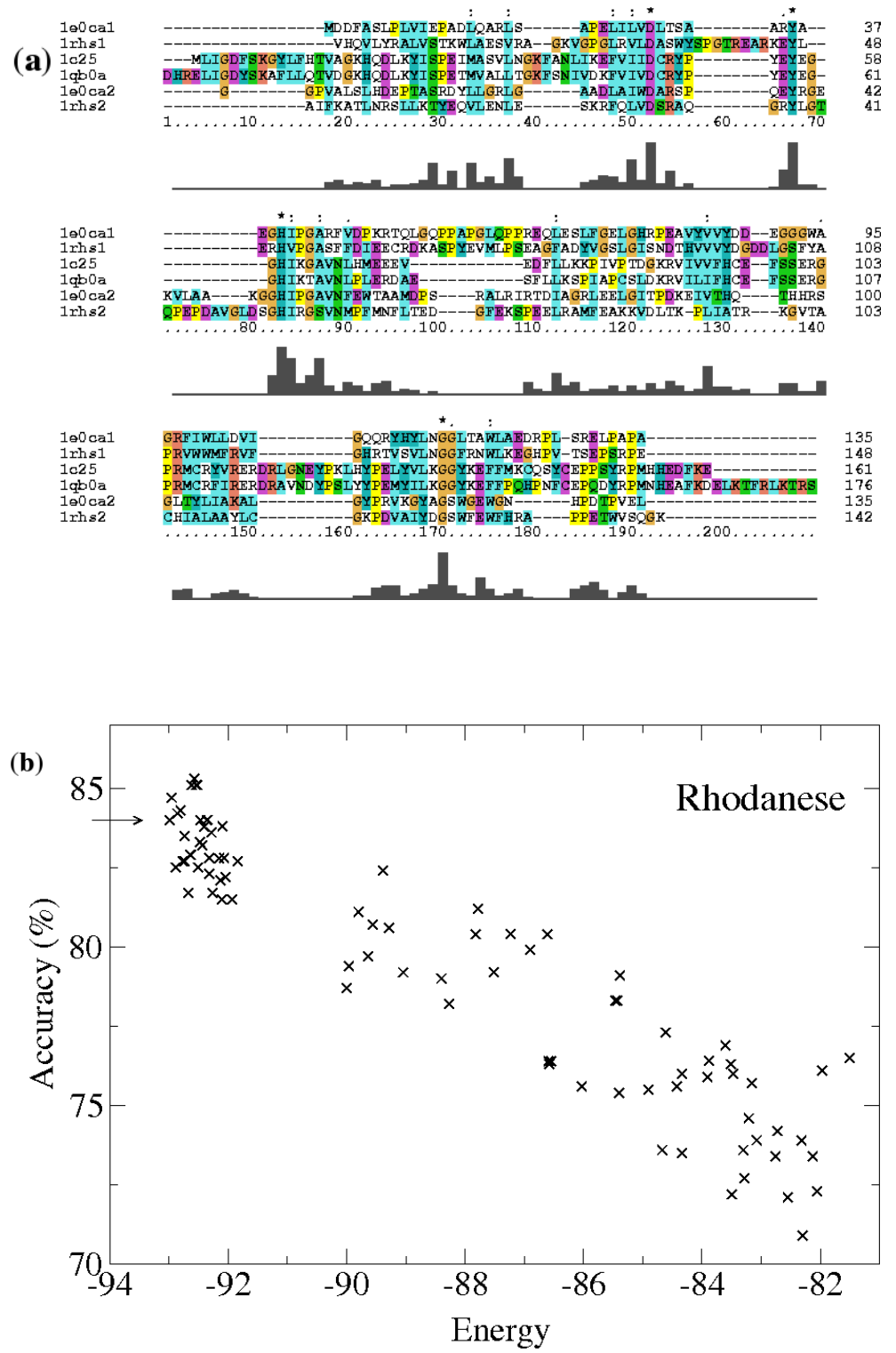

Fig. 2 An example of the lowest-energy multiple sequence alignment (a) and the energy landscape (b) of the alignment for Rhodanese family from the HOMSTRAD database is shown. The Rhodanese family consists of six structurally homologous proteins, and the level of sequence similarities is shown as a histogram in (a). Alternative alignments as well as the lowest-energy alignment are obtained by optimizing $E(A)$ of eq. (1) by MSACSA. Each symbol in the energy landscape represents an alternative alignment generated by MSACSA. The $x$-axis represents the value of $E(A)$, and the y-axis represents the alignment accuracy relative to the reference alignment constructed by human inspection of six protein structures. In (b), the lowest-energy alignment is indicated by an arrow, and it should be noted that it does not correspond to the most accurate alignment relative to the reference. Therefore, one should consider several low-energy alternative alignments to generate accurate protein models. Figure (a) is generated by clustalX program. 

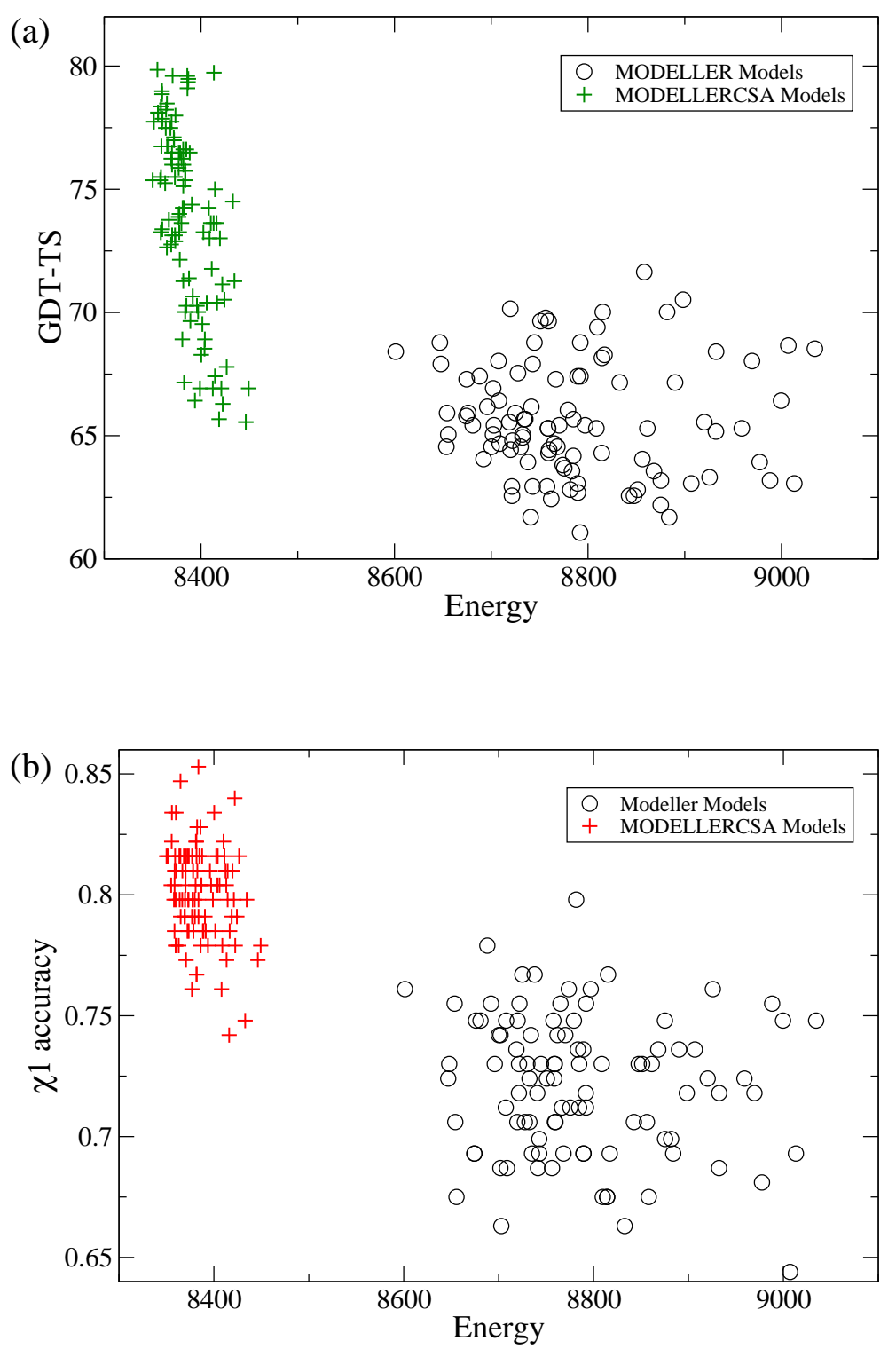

Fig. 3 Backbone accuracies (a) and side-chain accuracies (b) are plotted in terms of MODELLER energy for MODELLER generated models and MODELLERCSA generated models of sodfe family from HOMSTRAD database. The backbone accuracy is measured by GDT-TS, which is used in CASP assessment as a standard measure. The side-chain accuracy is measured by $\chi_{1}$, which is the percentage of correct rotamer within $30^{\circ}$ from the native structure. 


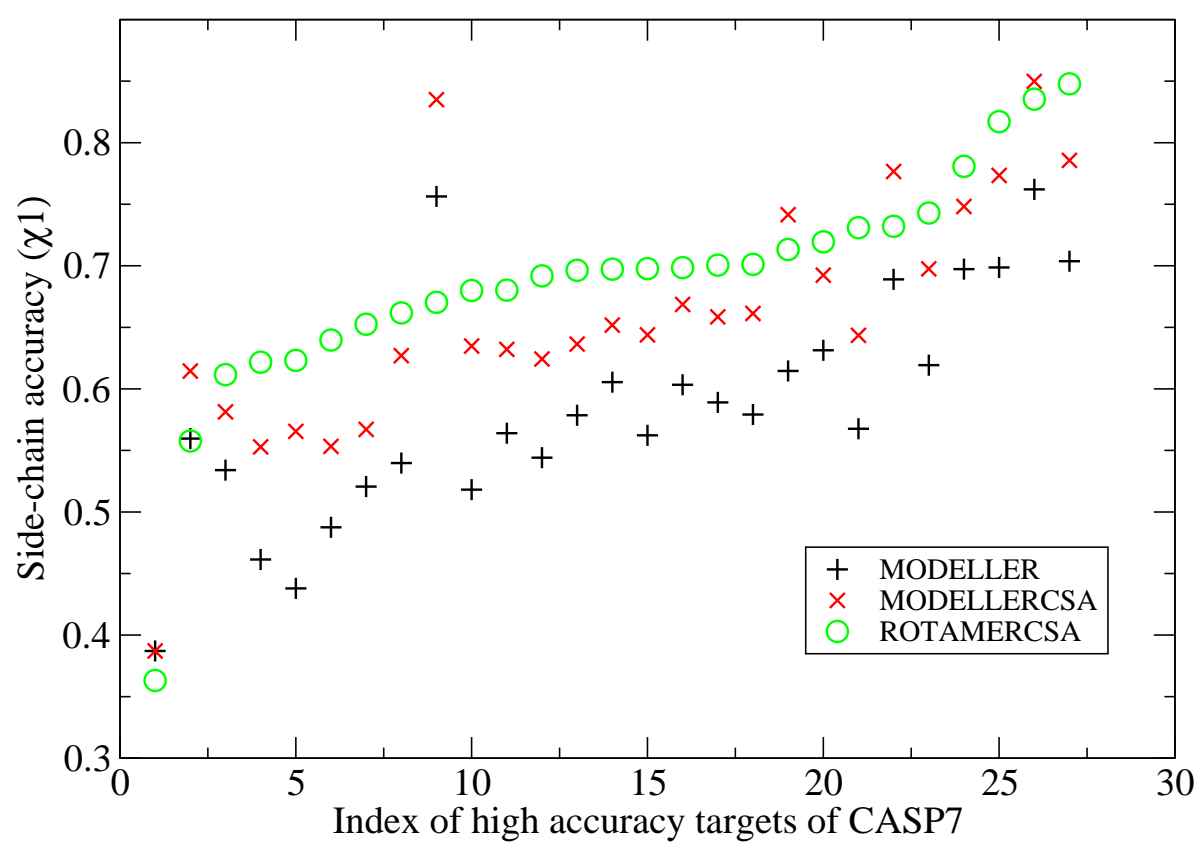

Fig. 4 Side-chain accuracies for 27 high accuracy TBM targets of CASP7 are shown. Roman cross symbols $(+)$ correspond to the models generated simply by executing MODELLER program. Crosses (x) correspond to the models obtained by MODELLERCSA. Circles (o) correspond to the models where backbones are kept identical to the MODELLERCSA results, and side-chains are remodelled by ROTAMERCSA. Overall side-chain accuracy improves gradually by applying more sophisticated methods than simple MODELLER chain building. Executing additional ROTAMERCSA after MODELLERCSA improves $\chi_{1}$ accuracy, although there are cases where best $\chi_{1}$ accuracy is achieved by MODELLERCSA ( 5 out of 27 ). 


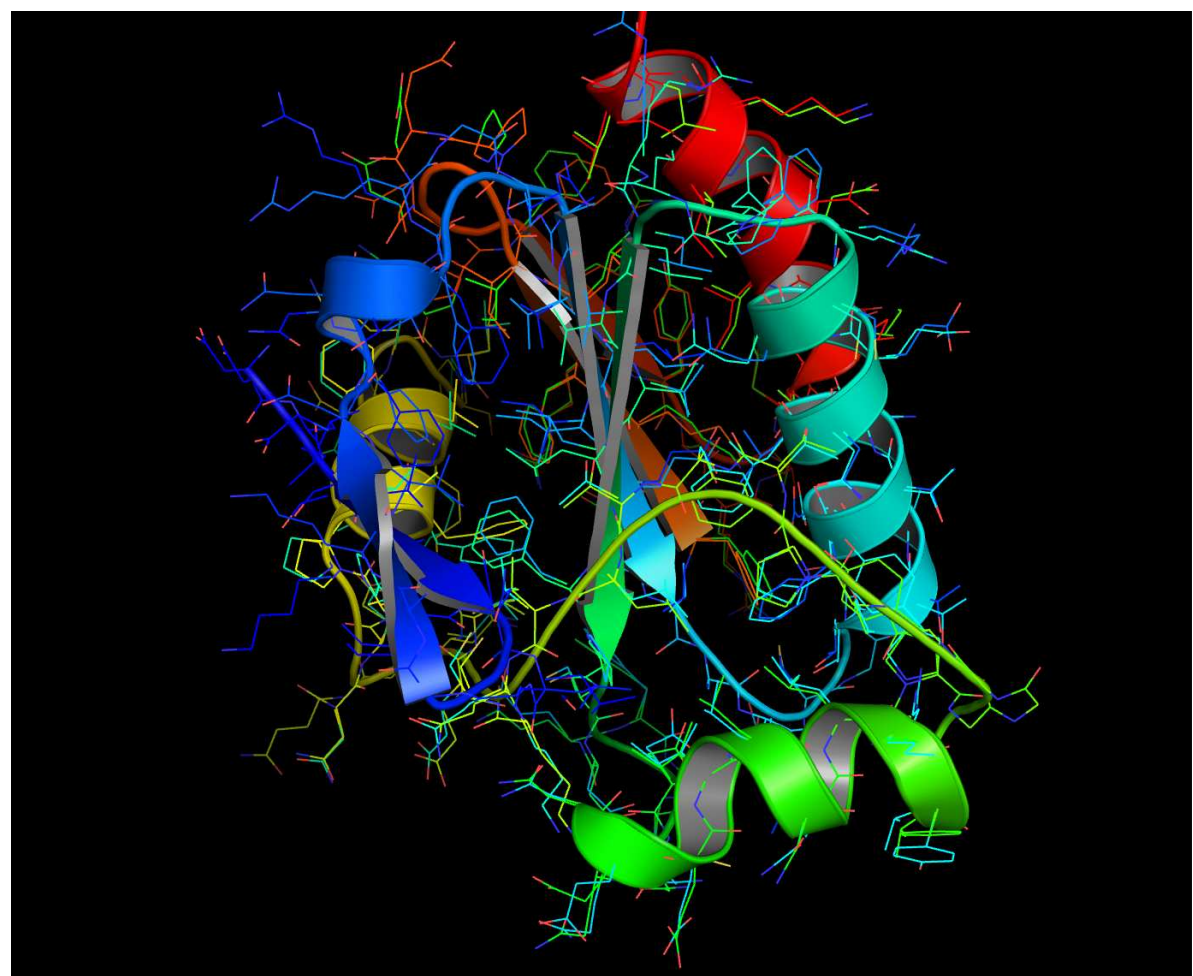

Fig. 5 The superposition between the native structure of T0345 (PDB ID: 2he3) and the lowest energy model generated by the full CASP7 procedure is shown. The model was constructed and submitted as the LEE model ( model 1 ) prior to the release of the native structure. Backbone heavy atom RMSD between the model and the native structure is about $1.6 \AA$ for the entire chain of 173 residues. The GDT-TS score is 96.0, The cartoon figures represent the native backbone structure and the model backbone structure, indistinguishable from each other. The $\chi_{1}$ angle accuracies are improved through the steps discussed in this chapter from the value of 70.4 (MODELLER), to 78.6 (MODELLERCSA) and finally to 84.8 (ROTAMERCSA). Aromatic residues in the core region are well predicted. Some exposed side-chains, especially lysine side-chains, do not agree between the two structures. The figure is generated by pymol. 